\title{
Maintenir l'élimination de la rougeole au Canada : Aller de l'avant
}

\author{
Lam $\mathbf{P}^{1}$, Williams $L^{1}$, Gadient $S^{1}$, Squires $S^{1}$, St-Laurent $\mathbf{M}^{1 *}$ \\ ${ }^{1}$ Centre de l'immunisation et des maladies respiratoires infectieuses, Agence de la santé publique du Canada, Ottawa \\ (Ontario)
}

*Correspondance : monique.st-laurent@phac-aspc.gc.ca

\section{Résumé}

Grâce à une immunisation réussie et à une surveillance appropriée, le Canada n'a signalé aucun cas de rougeole endémique depuis 1998. Cependant, des éclosions récentes et importantes attribuables à l'importation ont souligné les risques de réintroduction de la maladie par les voyages et des lacunes dans la couverture vaccinale des sous-populations.

Dans le prolongement de son rapport sur l'élimination de 2011 et en collaboration avec les partenaires provinciaux et territoriaux, l'Agence de la santé publique du Canada encourage l'immunisation en diffusant l'information recueillie dans les enquêtes sur la couverture vaccinale, en pilotant la surveillance accrue à l'aide de notifications en temps réel de cas suspectés et confirmés afin de permettre la détection précoce des éclosions, et en renforçant la capacité de ses laboratoires. À mesure que ces efforts sont renforcés, cette approche peut devenir un modèle pour les autres pays du monde cherchant à éliminer la rougeole.

\section{Introduction}

Le Canada lutte contre la rougeole endémique depuis que l'élimination de cette maladie est devenue un objectif national, lors de la Conférence de concertation de 1992 (1). La rougeole est éliminée lorsque la transmission indigène est interrompue pendant 12 mois consécutifs ou plus (2). Grâce au succès des programmes de vaccination systématique à deux doses et des campagnes de rattrapage, aucun cas de rougeole endémique n'a été signalé depuis 1998 (3). Cette réussite significative souligne l'effort de collaboration entrepris par tous les ordres de gouvernement, les fournisseurs de soins de santé, les organisations non gouvernementales, les intervenants experts et le public canadien.

Tant que la rougeole ne sera pas éradiquée à l'échelle mondiale, il faut s'attendre à ce que les cas importés de la maladie (c.-à-d. l'exposition survenue à l'extérieur du Canada) continuent. Depuis l'élimination de la rougeole, le nombre de cas est resté bas, avec une incidence médiane annuelle de 0,87 pour 1000000 d'habitants (données non publiées du Système canadien de surveillance de la rougeole et de la rubéole [SCSRR]). Cependant, ces dernières années, d'importantes éclosions se sont produites en raison d'importations dans des communautés où la couverture vaccinale est inadéquate, au Canada et dans d'autres pays ayant obtenu le statut d'élimination de la rougeole dans la Région des Amériques de l'Organisation mondiale de la Santé (OMS) (c.-à-d. aux États-Unis, au Brésil) (4).

Prenant conscience des défis que représente le maintien de l'élimination de la rougeole, l'Organisation panaméricaine de la Santé (OPS) a adopté une résolution au cours de la 150e séance du Comité exécutif de I'OPS pour mettre en œuvre un plan d'action d'urgence poussant tous les États membres à renforcer la surveillance de la maladie et la couverture vaccinale, afin de conserver leur statut d'élimination (5). Même si le Canada a réussi à contenir les éclosions précédentes, un effort accru est nécessaire pour favoriser l'élimination de la rougeole.

En 2011, l'Agence de la santé publique du Canada (l'Agence) a soumis à l'OPS le rapport de documentation et de vérification Élimination de la rougeole, de la rubéole et du syndrome de rubéole congénitale au Canada (6). Ce rapport présentait des preuves de l'élimination de la rougeole au Canada et fournissait des recommandations visant à conserver le statut d'élimination dans les domaines prioritaires, notamment : 1) la promotion de 
l'immunisation et le suivi de la couverture vaccinale ; 2) la surveillance et l'intervention en cas d'éclosion ; et 3) la capacité des laboratoires. L'Agence évalue les activités en cours dans chacun de ces domaines d'intérêt afin de renforcer l'approche du Canada sur le maintien de l'élimination.

\section{Promotion de l'immunisation et suivi de la couverture vaccinale}

Maintenir l'élimination de la rougeole nécessite une couverture vaccinale d'au moins $95 \%$ pour toutes les cohortes de population (7). Au Canada, les estimations nationales actuelles de la vaccination contre la rougeole pour les enfants indiquent une couverture adéquate : $95 \%$ des enfants reçoivent deux doses d'un vaccin contenant le virus de la rougeole avant l'âge de sept ans (8). Cependant, la couverture vaccinale aux niveaux provincial, territorial et local est hétérogène car dans certaines sous-populations, l'adoption de la vaccination est sous-optimale ou la vaccination est refusée. L'éclosion en Colombie-Britannique de 2014 (9) et la récente éclosion au Québec début 2015 (4) sont des exemples de rougeole importée dans une communauté où la vaccination a été refusée pour des raisons religieuses ou philosophiques. Les personnes refusant tous les vaccins ne représentent qu'une petite partie des réticents face à la vaccination (10). Certains parents peuvent retarder la vaccination de routine de leurs enfants ou ne choisir que certains vaccins, ce qui rend certaines personnes vulnérables aux infections (10).

Le Groupe stratégique consultatif d'experts (SAGE) et le Groupe de travail d'immunisation sur la réticence face à la vaccination recommande aux pays d'intégrer aux activités de leur programme d'immunisation un plan pour mesurer et remédier à la réticence face à la vaccination (11). Dans le cadre de la Stratégie nationale d'immunisation du Canada, un Groupe de travail expert sur l'acceptation et l'adoption des vaccins a été formé pour répondre aux problèmes liés à l'accès aux vaccins et à leur adoption, afin d'améliorer les taux de couverture vaccinale au Canada. L'Agence continue de promouvoir l'immunisation en distribuant au public des documents éducatifs et en soutenant des initiatives novatrices comme ImmunizeCA, une application mobile de vaccination gratuite permettant aux Canadiens de suivre facilement leurs dossiers de vaccination (12).

En l'absence d'un réseau national de registres d'immunisation, il est nécessaire de mener des enquêtes périodiques auprès de la population afin de vérifier que la couverture vaccinale nationale est adéquate. L'Agence surveille la couverture vaccinale contre la rougeole au moyen de l'Enquête sur la couverture vaccinale nationale des enfants (ECVNE) (8). Le modèle du cycle d'enquête de 2013 a été généralisé afin de fournir des estimations aux niveaux provincial et territorial. La phase de validation des fournisseurs de soins de santé a pris fin en 2014 et les résultats de l'enquête devraient être publiés plus tard cette année. Les connaissances, les attitudes et les comportements parentaux par rapport à la vaccination sont également pris en compte par l'ECVNE afin de mieux comprendre les facteurs qui influencent les décisions en matière d'immunisation.

Un réseau national de registres d'immunisation permet au Canada de planifier et de cibler les efforts d'immunisation, d'éviter tout dédoublement inutile des vaccinations et de faciliter les interventions en cas d'éclosion. L'Agence continue de soutenir le Réseau canadien des registres d'immunisation et d'autres organisations de normalisation afin de promouvoir les normes nationales pour les registres d'immunisation et de faciliter le transfert de dossiers de vaccination électroniques entre les registres. Les normes nationales pour l'évaluation de la couverture vaccinale sont publiées sur le site Web de l'Agence (13). Les normes de codes à barres pour les produits de vaccination et les normes fonctionnelles pour les registres d'immunisation seront respectivement disponibles en 2015 et d'ici la fin 2016.

\section{Surveillance et intervention en cas d'éclosion}

La détection précoce au moyen d'activités complètes de surveillance est un élément clé permettant de gérer les cas importés et d'assurer une intervention rapide et efficace en cas d'éclosion. Entre 1998 et 2014,

159 importations de rougeole ont été observées en provenance de 37 pays différents, et seulement $28 \%$ des cas importés ont entraîné une transmission secondaire (données non publiées du SCSRR). Les cas de rougeole sont principalement enregistrés par le SCSRR à travers des rapports hebdomadaires de provinces et de territoires (14).

Le projet pilote de l'Agence sur la surveillance de la rougeole et de la rubéole (MARS) est un système en ligne fournissant des notifications en temps réel de cas suspectés et confirmés. Depuis 2011, le MARS a été mis en œuvre dans trois juridictions (Colombie-Britannique, Alberta et Terre-Neuve). II offre une plateforme permettant 
d'établir des liens entre les données épidémiologiques et les données de laboratoires provenant du laboratoire provincial, du bureau de santé publique provincial et des laboratoires médicaux nationaux partenaires. Dans le cadre des priorités de l'Agence visant à augmenter l'accès en temps réel aux renseignements en matière de santé publique, le MARS sera proposé à d'autres juridictions fin 2015.

Si les éclosions de rougeole sont rares dans l'ère de post-élimination, des éclosions plus importantes peuvent être un énorme fardeau pour les ressources de santé publique. Il y a un manque de données sur les coûts associés au contrôle des éclosions de rougeole au Canada et les estimations ont été limitées. En 2014, une éclosion de quatre cas de rougeole à Ottawa a nécessité la participation de 60 employés de la santé publique, qui ont cumulé 1400 heures de travail (15). Cela a causé un déficit de $28000 \$(C A)$ dans le budget du premier trimestre du bureau local de santé publique.

L'Agence fournit des conseils aux provinces et aux territoires en finançant les enquêtes et le contrôle des éclosions. Dans l'optique de fournir les meilleures données probantes accessibles, l'Agence a collaboré avec l'Agence canadienne des médicaments et des technologies de la santé (ACMTS) pour mener un examen systématique des interventions afin de réduire la propagation secondaire de la rougeole (17), ainsi qu'une analyse des coûts pour estimer l'utilisation des ressources associées aux éclosions de rougeole (18).

\section{Capacité des laboratoires}

La surveillance moléculaire et le génotypage (processus consistant à déterminer les différences dans la composition génétique d'un organisme) sont essentiels pour comprendre l'épidémiologie de la rougeole au Canada. Ce sont des outils inestimables pour les enquêtes de santé publique visant à confirmer les antécédents d'exposition, à établir des liens entre les éclosions simultanées et à identifier de potentielles sources d'importations (19). En outre, ils permettent de vérifier le statut d'élimination du Canada en faisant la distinction entre différentes variantes d'une souche et la propagation étendue d'une seule souche.

À mesure que nous progressons vers l'éradication de la rougeole au niveau mondial, la diversité génétique du virus va diminuer. Ainsi, il deviendra de plus en plus difficile de distinguer plusieurs importations d'une transmission endémique lorsque des cas sporadiques auront la même séquence génétique. II faudra avoir plus fréquemment recours au génotypage étendu afin d'assurer une surveillance adéquate.

Le Laboratoire national de microbiologie (LNM) de l'Agence est un laboratoire régional de référence pour la rougeole désigné par l'OPS et l'OMS. II est responsable du génotypage et de la surveillance moléculaire accrue de tous les échantillons de rougeole au Canada. Le LNM continue à explorer des méthodes novatrices visant à accroître le séquençage du virus de la rougeole afin de fournir des résultats en temps opportun pour faciliter les enquêtes sur les éclosions.

\section{Conclusion}

En raison d'une activité de la rougeole dans d'autres régions du monde, le statut d'élimination du Canada va continuer d'être mis au défi, ce qui entraînera sûrement des éclosions limitées comme celles qui se sont récemment produites en Colombie-Britannique (9) et au Québec (4). La menace des importations, combinée à la couverture vaccinale sous-optimale dans les sous-populations, présente un risque de réintroduction de la maladie. Pour que le Canada puisse maintenir l'élimination de la rougeole, il est nécessaire d'accroître les programmes actuels et de continuer la collaboration à tous les ordres de gouvernement. Dans le prolongement des recommandations de son rapport sur l'élimination de 2011 (6), l'Agence encourage l'immunisation en diffusant l'information recueillie dans les enquêtes sur la couverture vaccinale, en pilotant la surveillance accrue à l'aide de notifications en temps réel de cas suspectés et confirmés afin de permettre la détection précoce des éclosions, et en renforçant la capacité de ses laboratoires. À mesure que ces efforts sont renforcés, cette approche peut devenir un modèle pour les autres pays du monde cherchant à éliminer la rougeole. 


\section{Conflit d'intérêts}

Aucun

\section{Financement}

Po-Po Lam est financée par une bourse de politiques scientifiques attribuée par les Instituts de recherche en santé du Canada (IRSC).

\section{Références}

(1) Consensus Conference on Measles. CCDR. 1993 May 30;19(10):72-9.

(2) Papania MJ, Orenstein WA. Defining and assessing measles elimination goals. J Infect Dis. 2004 May 1;189 Suppl 1:S23-6.

(3) King A, Varughese P, De Serres G, Tipples GA, Waters J, Working Group on Measles Elimination. Measles elimination in Canada. J Infect Dis. 2004 May 1;189 Suppl 1:S236-42.

(4) Pan American Health Organization, World Health Organization [Internet]. Epidemiological alert. Measles outbreaks and implications for the Americas. 9 February 2015. Washington DC; PAHO; 2015.

http://www.paho.org/hq/index.php?option=com_docman\&task=doc_view\&Itemid=270\&gid=29016\&lang=en.

(5) Pan American Health Organization. 150th session of the Executive Committee. Washington DC: Pan-American Health Organization/World Health Organization; 2012.

http://www.paho.org/hq/index.php?option=com_docman\&task=doc_download\&gid=17573\&ltemid=.

(6) Public Health Agency of Canada. Elimination of measles, rubella and congenital rubella syndrome in Canada: Documentation and verification report. Ottawa ON: Public Health Agency of Canada; 2015.http://www.phacaspc.gc.ca/im/vpd-mev/measles-rougeole-mrer-eng.php.

Disponible en français: http://www.phac-aspc.gc.ca/im/vpd-mev/measles-rougeole-mg-fra.php

(7) Pan American Health Organization. Plan of action for the documentation and verification of measles, rubella, and congenital rubella syndrome elimination in the Region of the Americas. Washington DC: Pan American Health Organization; 2015. http://www.paho.org/hq/index.php?option=com_docman\&task=doc_download\&gid=16739.

(8) Public Health Agency of Canada [Internet]. Vaccine coverage in Canadian children: Results from the 2011 childhood national immunization coverage survey. Ottawa ON: Public Health Agency of Canada; 2015. http://www.phacaspc.gc.ca/im/nics-enva/vccc-cvec-eng.php.

Disponible en français : http://www.phac-aspc.gc.ca/im/nics-enva/vccc-cvec-fra.php

(9) Naus M, Puddicombe D, Murti M, Fung C, Stam R, Loadman S et al. Outbreak of measles in an unvaccinated population, British Columbia, 2014. CCDR. 2015 Jul 2;41(171-177).

(10) MacDonald NE, SAGE Working Group on Vaccine Hesitancy. Vaccine hesitancy: Definition, scope and determinants. Vaccine. Vaccine. 2015 Apr 172015.

(11) Eskola J, Duclos P, Schuster M, MacDonald NE, SAGE Working Group on Vaccine Hesitancy. How to deal with vaccine hesitancy? Vaccine. 2015 Apr 18.

(12) Immunize Canada [Internet]. ImmunizeCA App. Ottawa: Immunize Canada; 2014 http://www.immunize.ca/en/app.aspx

(13) Public Health Agency of Canada [Internet]. National standards for immunization coverage assessment: Recommendations from the Canadian immunization registry network. Ottawa ON: Public Health Agency of Canada; 2015 http://www.phac-aspc.gc.ca/im/nationalstandards-normenationales-eng.php Disponible en français : http://www.phac-aspc.gc.ca/im/nationalstandards-normenationales-fra.php

(14) Government of Canada [Internet]. Surveillance of measles. Ottawa ON: Government of Canada; 2015. http://www.healthycanadians.gc.ca/diseases-conditions-maladies-affections/disease-maladie/measlesrougeole/surveillance-eng.php.

(15) Greenway T. Measles outbreak causes Ottawa public health to run early deficit.Metro News. 2014 May 6. http://metronews.ca/news/ottawa/1024794/measles-outbreak-causes-ottawa-public-health-to-run-early-deficit/. City of Ottawa. 2014 operating budget Q1 - Board of health for the city of Ottawa health unit status report. Ottawa ON: City of Ottawa; 2015. http://ottawa.ca/calendar/ottawa/citycouncil/obh/2014/05-05/Q1\%20Budget\%20Report.pdf. Foerster V, Perras C, Spry C, Weeks L. Public health interventions to reduce the secondary spread of measles. CADTH rapid response report: Systematic review. Ottawa ON: CADTH; May 2015. https://www.cadth.ca/publichealth-interventions-reduce-secondary-spread-measles

(18) Budden A, Lee KM, Lam P. Costs of contact tracing activities aimed at reducing the transmission of measles in Canada. CADTH rapid reponse report: Systematic review. Ottawa ON: CADTH; June 2015.

https://www.cadth.ca/costs-contact-tracing-activities-aimed-reducing-transmission-measles-canada

(19) Heibert J, Severini A. Measles molecular epidemiology: What does it tell us and why is it important? CCDR. 2014;40(12):257.

Disponible en français : http://www.phac-aspc.gc.ca/publicat/ccdr-rmtc/14vol40/dr-rm40-12/index-fra.p 